will act as a foreign body and not be sequestrated. This is not true of any other bone infection in the body. For example, one half of a long bone may be devitalized through infection and be thrown off as a sequestrum. There can be no organic union between a tooth root which has lost its peridental membrane and the surrounding bone or soft tissue. It would seem, therefore, that these elemental fundamental facts must be our guide in undertaking the management of infected teeth.

Dr. E. S. Fuller, Dayton, Ohio: Some years ago, in order to obtain better access for cleaning the root surface of a lower incisor, I made an incision along the axial line of the tooth, cleaned the surface and sutured the gum flaps together. The stutures pulled out and the root surface became exposed. This I thought unfortunate at the time, but now I think it was the best thing which could have happened. During the past three years I have been treating these "pyorrhea pockets" by removing the overlying gum tissue in all cases, excepting those where the pockets were shallow enough to become obliterated by the reduction of the gingival inflammation following the removal of deposits and the cleaning of the root surface. Many cases are seen showing a deep "pyorrhea pocket" on both the mesial and distal proximal surfaces of a tooth-say a bicuspid, for example-the adjoining teeth not being affected. In these cases I frequently extract the diseased tooth rather than resect away the gum tissue from the interproximal space, as a horizontal resection would destroy the attachment of the tooth not involved.

Dr. Arthl一 Zextler, New York: To understand the character of the union obtained we would have to secure a section embracing all the tissues concerned and submit the whole for histologic examination. It is not easy to obtain human subjects for this. Animal experimentation may be used for the purpose. I believe, however, that we do not have the same conditions in animal as in human mouths. Practical examination shows a union, in operated cases, difficult to penetrate with fine probes. Only by exposing the entire involved area can one eradicate the disease. Simply cutting away the pocket has not proved permanent.

Where I operate at the same time for peridental and periapical infection, I make the incisions as described in my operation. A month after removing the sutures there is no sign left of these incisions, while with the semicircular incisions for apicoectomies cicatrices are present for a long time. One need not fear to suture the flap in either of these operations, provided the operation is done as aseptically as it should be done. I never had an ulterior infection due to suturing. I thought it understood that only healthy tissue is allowed to remain, and in speaking of reattachment $\mathbf{I}$ did not mean a reattachment of pericementum, but a reattachment of soft tissues that had been lifted and then replaced carefully, saving the healthy periosteum. Twice I operated in cases previously splinted. The operations were failures. It was due, I believe, to the lack of normal motion. It interferes with circulation and deprives the part of proper nutrition.

It is true that the fundamental pathology is with the hard tissues and not with the soft, and that is precisely why I felt the necessity of lifting a flap and of getting to the hard tissues underneath. That is why I chisel away a strip of the involved alveolar plate as well as curetting the soft involved tissue which lies over it and surrounds the roots. That is why I pronounced it a poor operative risk to operate when all investing tissue is gone, and advise in such cases removal of teeth.

As to the general condition of the patient, I think it is understood that if it cannot be controlled one need not attempt local treatment expecting success. Both must go hand in hand. The local circulation will be improved by the operation if we remove the infected parts. The general circulation must be taken care of by the patient's physician.

Dr. Brophy: Is there not some vitality left in the cementum?

Dr. ZentLeR: Wherever there is cementum left on a portion of the root that had prior to operating a detachment of the investing tissue, it is an open question in my mind whether this cementum is not able to throw off further cells into newly created healthy surroundings to form some sort of attachment. Where suppuration has intervened I do not believe there will be any live cementum. We get a reattachment of healthy tissue to healthy tissue in approximating them. You need not fear at all to remove some of the healthy tissue if you must do it in order to eradicate all the diseased tissue. The lost healthy tissue will be replaced the same as in any other surgical procedure.

\section{POSSIBILITIES OF FRACTIONAL GASTRIC ANALYSIS *}

\section{MARTIN E. REHFUSS, M.D. PHILADELPHIA}

Fractional gastric analysis has for its purpose the determination of the evolution of gastric digestion. The necessity for such extended technic is justified by the fact that gastric digestion is marked by a series of constantly changing phases, so that it may now be definitely stated that an examination of any single phase of the digestive curve gives no information as to what has preceded or what will follow that phase. The most marked changes may occur in comparatively short intervals, so that findings at one period may be doubled or tripled at another period. The main point, however, is the essential fact that gastric digestion is the response of the organ to a definite stimulus, which, in health, evolves in a perfectly methodical and coordinated manner, and in disease evolves with every possible variation. In order, therefore, to interpret gastric disease, we must study the elements governing the response of the organ in health.

\section{NORMAL DIGESTION}

From a study of normal digestion with every form of foodstuff, which the entire staff of the chemical department of the Jefferson Medical College was engaged, we arrived at the following conclusions:

Human gastric digestion is divided into a series of recurring cycles which, for the want of better names, we have called the digestive and interdigestive cycles. These succeed each other in health with regularity and represent most perfectly the normal gastric mechanism. The digestive cycle is that portion in response to the ingestion of food of any kind and evolves in a perfectly coordinated manner. There is practically no latent period and there are well marked psychic and chemical phases to secretion. The psychic secretion is very considerable, as we have been able to show, and in health it probably averages 250 c.c., lasting from sixty to eighty minutes and being marked, according to our recent studies, by changes in environment, fatigue, etc. The total acidity averages 97.2 in our series and it is markedly reduced, but does not disappear with atropin administration. The chemical secretion probably cómmences early but reaches its height later and completes digestion. During this active period, we notice (1) the relaxation of the perisystole and the inauguration of peristalsis, and (2) a change from the resting secretion to a pronounced secretion of much higher acidity. Furthermore, we can detect certain regulating mechanisms such as tryptic or duodenal regurgitation regulating acidity. This cycle is constantly changing as each factor comes into play. It is followed by the interdigestive phase characteristic of normal digestion.

* Read before the Section on Gastro.Enterology and Proctology at the Sixty-Ninth Annual Session of the American Medical Association, Chicago, June, 1918 
The stomach is never empty, and there is always physiologically active secretion, but the resting secretion or the residuum seen in the interdigestive phase is very different from the digestive secretion. The quantity averages 50 c.c., the total average acidity being only 30 and the free acid 18 , while the presence of bile is much more constant ( 55 per cent) ; and tryptic regurgitation is almost constant. Instead of peristalsis, we have perisystole, tonal, and hunger contractions and a relaxed pylorus. It is the study of these phases so characteristic in health and altered by disease that yields us accurate information regarding gastric disease. All the motility changes only translate themselves by a diminution or increase in the interdigestive period.

We are now prepared to say that there is no one form of curve seen in health, but that the curve of the digestive period may be one of three kinds, depending on the type of individual, hypersecretory or hyposecretory. Further study of healthy persons only emphasizes the necessity for retaining these types. In a résumé of 842 complete curves on various foodstuffs with more than 20,000 titrations, we found that 383 , or 45 per cent., excecled 100 total acidity, and on that study embracing three years' work, we are prepared to state that no acid figures occurred in disease which could not be duplicated in health. In other words, we found that 45 per cent. of all responses in health showed so-called hyperacidity, while 42 per cent. of my ulcer series showed the same thing. In other words, there is no greater incidence of high acid figures in tulcer or in any other gastric disease than in health, a fact that raises the extremely important question as to whether an actual demonstrable hyperacidity ever does occur. We must face these facts squarely before we can make progress in this line. They are facts which may be verified and our ideas must be altered to meet them. Furthermore, we are prepared to state that hypersecretion in some form occurs in about 40 per cent of normal persons.

\section{PATHOLOGIC VARIATIONS}

In disease, every variation occurs from a total suppression of the gastric secretion to an exaggeration of all the phases. We might classify the elements into (1) those which are a modification of normal total acidity and free acid of the secretion, showing the presence of duodenal regurgitation, and (2) those which are frankly abnormal, such as blood, pus, mucus, certain organic acids, and pathogenic organisms constantly present. The study of normal digestion emphasizes one important point, namely, that at certain phases certain acidities and quantities of secretion are normal and at other phases these same figures are abnormal. For instance, instead of a coordinated curve after an Ewald meal with its height at the one hour and one and one-quarter hour point, we may have a total displacement of the curve, either a markedly exaggerated phase during the first hour or a slow initial phase, followed by pronounced findings at the end of the second hour. Study of pathologic curves shows that every variation of secretion may occur, instead of perfect evolution of digestion. There may be (1) a delay in digestion, (2) an acceleration in digestion, (3) a disturbance in secretory velocity resulting in hyposecretion or hypersecretion, and (4) alteration of digestion by the addition of frankly pathologic products, such as blood, pus and mucus.
We recognize that alterations may come through the systemic circulation (soluble toxins, bacteria) blood dyscrasias, resulting in altered mucosal conditions and altered secretory digestion, or through disturbances in the portal circulation (cirrhosis). These systemic conditions may stimulate or depress secretory activity. Again we know definitely that a lesion elsewhere in the gastro-intestinal tract (gallbladder, appendix) may increase the irritability of the vagus, inducing the secretory manifestations of vagotonia. In chronic gastritis we recognize as operative not merely the inability of the mucosa to form a complete secretion, but also the mechanism of neutralization of the secretion by the mucus. In ulcer we do not look for pathognomonic curves, for we realize that a nonobstructive ulcer gives a very different picture from pyloric stenosis with ulceration. In all forms there is a tendency toward vagotonia, pyloroplasm, hypersecretion, shortening of the interdigestive period, and increase in protein content. In duodenal ulcer, the most characteristic finding is that of positive blood at the phases of tryptic regurgitation. In a large group of duodenal ulcer cases there is present a late hypersecretion, accompanied by periodic regurgitation of duodenal material giving occult blood reaction. Gallbladder disease gives clean digestion and often high acidity without mucus, pus or blood, and when there is pericholecystitis, with adhesions to the duodenum, the adhesions closely resemble stenotic ulcer at the pylorus. However, there is a group of old gallbladder cases associated with duodenitis, in which a low curve with all the findings of true gastric infection may be detected. Appendicitis is most frequently accompanied by clean digestion, with high figures indicative of vagotonia. Cancer has as its characteristics the uniform and constant depression of secretory activity, together with the presence of its specific products, pus, blood, mucus, lactic acid, soluble protein, etc., each of which plays a part in the composition of the curve that is formed. These facts must be borne in mind. Nerve factors, circulatory toxins, the lack of building material, and direct local disease of the mucous membrane may all produce low acid curves, but they produce the curves very differently. The first and second each give a clean subacid curve, and the third is accompanied by elements such as mucus, pus and blood, which give a clue to its source. Let me illustrate: We can see in a certain anemia a low curve without any mucus, blood or pus; it is simply a subacid curve in anemia. The subacid curve in chronic gastritis is punctuated by the periodic secretion of quantities of mucts. In infectious gastritis, there is not merely mucus, but bacteria, pus and blood, and the same is true in case of carcinoma. Pericholecystitis with adhesions to the duodenum may give the same picture as contrasting pyloric ulcer, but blood and increased protein in the latter serve to distinguish it. We know that gallbladder disease, appendicitis, pancreatitis, intestinal adhesions and pelvic disease may all give reflexly vagotonia and the same gastric picture. It is the correlation of all the data which enables us to make the correct interpretation.

Some factors are important. The amino-acid content seems to run parallel to the curve of tryptic regurgitation. Pancreatic ferments or pancreatic regurgitation occurs independently of biliary regurgitation, particularly in the digestion of carbohydrates. With fats, both are likely to regurgitate. 
In the analysis of blood, we attach great significance to the position and type of bleeding. Blood synchronous with periods of active tryptic regurgitation is characteristic of duodenal ulcer. Blood throughout the entire digestive phase of subacid cases is seen particularly in cancer and infected gastritis. Blood in the interdigestive period and not in the digestive cycle is likely to be swallowed blood. With pus we must distinguish between extragastric or swallowed pus and intragastric pus or that formed in the gastric wall. The former is always seen in the interdigestive cycle and is diagnostic; the latter is seen in both cycles.

The problems that confront us in the interpretation of gastric work are legion. Fractional analysis only emphasizes how many there are and offers a method for their solution. In the normal subject, we can produce high acid curves with meat, milk, low acid curves with fruit and vegetables, and we can produce an experimental subacidity or achylia with concentrated salt or glucose solution, or we alter the vagus and the sympathetic tonus by drugs and ductless gland preparations that alter the gastric response. Bacterial, chemical, neural, circulatory, and local mucosal disturbances can alter the gastric output, but only actual local organic disease can produce pus, blood, mucus and soluble protein bacteria.

Fractional gastric analysis already has an enviable record. It has emphasized the importance of the digestive and interdigestive cycles. It has enabled us to study the true residuum and to isolate and easily to diagnose swallowed suppurative material from infected processes higher up. It has enabled us to trace exactly the trend of digestion and depict the factors occasioning disturbance. It has opened up new chapters on psychic secretion, automatic regulation of acidity by tryptic regurgitation, and protein curves, and it has emphasized the fact that no one can guess the nature of gastric work but only painstaking technic will reveal all its details.

\section{TOXINS AS DEPRESSANTS}

Toxins of a bacterial nature usually act as depressants to the secretion. There are certain infections in the nose and throat, however, which unquestionably induce increased ragus tone and result in an increased secretion. In the analysis of the gastric curve, many points must be taken into consideration. Anomalies in the gastric secretion may be simply a perversion in secretory output but without the addition of evidence of local disease. For instance, gallbladder, appendiceal, intestinal, pancreatic and nervous conditions may give rise to well marked changes in the curve, either a prolongation of digestion, late hypersecretion, or other changes. But as soon as we have the addition of mucus, pus, blood, or bacteria, we are probably dealing with a pathologic process in the stomach itself.

Take, for instance, the question of delayed digestion, in which evolves a low, slow curve that is clearly pathologic. This curve is encountered in cases of chronic gastritis, late pulmonary tuberculosis, a group of anemias, chronic parenchymatitis, nephritis, a small group of old ulcers, and finally in a very large group of gastric cancer. In each case the mechanism is different, although in all there is the underlying disturbance in the nucous membrane, possibly primary in certain forms of gastritis and carcinoma, and clearly secondary in anemias, nephritis, hepatic disorders, cirrhosis and tuberculosis. In the cancer group, we see a disproportionate reduction in free acid and the presence of pus, blood, mucus, lactic acid, etc. In chronic gastritis we see simply a disproportionate amount of mucus. In some of the anemias and in nephritis there may be no increase in mucus, and free acid runs proportionate or parallel to total acidity. A cure may follow local treatment, it may follow treatment directed to the heart or kidneys, or again, curiously enough, these secretory delays may suddenly clear up after removal of a focal infection in the teeth, the sinuses, the tonsils, etc. I have case records in which the entire response was altered after such procedure. The whole problem is so complex that painstaking research alone will solve it.

We have been able to show that the delayed curve of acidity may come about (1) through a delayed elaboration of secretion on the part of the mucous membrane, (2) through a strong duodenal regurgitation neutralizing an apparently normal secretory output, and (3) through the neutralization of the secretion by pathologic products, such as mucus, pus and blood. The first form is accompanied by no evidence of either tryptic regurgitation, or mucus, pus and blood, and may be due to anemias or to circulating toxins, or may be the result of disease elsewhere altering the circulation of the nervous supply. The second form is shown by the evidence of regurgitation in laboratory study. The third form belongs to the group of true gitstric infections and cancer. All of them are susceptible to careful analysis.

\section{CONCLUSIONS}

1. Fractional gastric analysis alone can reveal the evolution of gastric digestion. Gastric digestion, whether normal or pathologic, consists of a series of constantly changing phases. Certain phases are normal, certain are obviously pathologic.

2. Normal gastric physiology consists in a rhythmic series of cycles in which we may differentiate the digestive and the interdigestive periods. In health, they succeed each other regularly; in disease, their sequence is seriously interfered with.

3 . The digestive cycle is the response of the stomach to a definite stimulus. The response is partly psychic and partly chemical, but in its essential characteristics, it differs from the interdigestive phase.

4. There is always a physiologically active secretion in the stomach but the characteristics of the two periods are very different. The digestive secretion persisting into the interdigestive period is pathologic.

5. Our studies of healthy men indicate that there is no acidity in disease which cannot be encountered in health, and the diagnostic value of high acidity must be very carefully worked out. Forty-five per cent. of more than 800 cases (normal) gave a total acidity of 100 in excess. Normally, we point out three different secretory types.

6. Pathologically every possible variation in secretory output occurs. At present it seems that there are many possibilities in the analysis of each varieiy of curve. It is at this point that circumstantial evidence by the study of the protein curve, pus, blood, mucus, bacteria and tryptic regurgitation may throw light on the subject. In every case the extragastric as well as the gastric causes must be reviewed.

7. The gastric response may be shortened, delayed or exaggerated. It may be accompanied by the most 
marked variations in secretory and acid concentration output. It may be altered by the addition of pus, blood, mucus and bacteria in every possible way, and these variations may occur only at certain portions of the curve, emphasizing the necessity for complete examination.

8. The investigation of psychic secretion, tryptic and biliary regurgitation, hypersecretion, protein concentration and secretory velocity, and of the evidence of pathologic material is full of promise.

\section{ABSTRACT OF DISCUSSION}

Dr. A. F. R. ANderson, Brooklyn: A study of findings on fractional examination of gastric contents in about 800 patients leads to the following conclusions: The only curve of acidity which is of itself of absolute value in diagnosis is that found in achylia gastrica, a straight base line, representing free hydrochloric acid, running along the zero line, and a slightly varying curve of total acidity. The achylia of malignancy is quite often found to be associated with a high total acidity. The reflex type of curve is also of considerable value as indicating an inflammatory condition somewhere in the abdomen or pelvis, causing a reflex manifestation in the stomach. A finding which we consider typical of duodenal ulcer is the occurrence of blood and bile coincidently during the fractional examination. Trauma from the tip of the tulse catching in the pylorus and being pulled on must be excluded, but sufficient trauma to cause bleeding is exceedingly rare. Pyloric chstruction will show the normal overnight residue and the isual findings of carcinoma or of ulcer. Aiter gastro-enterostomy for ulcer, bile is usually present in considerable amount, and when blood is also present it may mean persistence of the original ulcer, or the presence of a jejunal ulcer. Gastric motility can be determined more accurately by the fractional method than by usual routine roentgen-ray examination. Cases of gastroptosis showing a normal or even a slightly increased motility by the test meal examination often show much residue six hours after the usual sized barium meal. When, subsequently, a smaller barium meal is given, normal emptying may occur.

Dr. William Van Valzah Hayes, New York: The practical question that comes up is, How can we, as gastro-enterologists, employ this sort of technic in the ordinary case? There ate many pros and cons on the subject, and up to date 1 have not used the method very frequently. In the ordinary case, in addition to a careful history and careful physical examination, examination of the stool, etc., we want to find out what the stomach does in the early and in the late stages of digestion. Are we going to put in a tube which will make the patient uncomfortable, which will cause a continuous flow of mucus and other secretions, and more or less obscure certain phases of the gastric activity, or can we, with less trouble to the patient and to ourselves, get the data we need? I believe that in the average case we can get these data by simply giving the test breakfast and taking it out an hour later. Then the following day give a duration test, i. e., two soit boiled eggs, a roll and a glass of water. Three and a half hours later use the tube to see whether the stomach has cmptied itself as it should have, or whether there remains a considerable amount of gastric contents, with high acidity and possilly regurgitated blood or bile from the duodenum. Diagnosis is nearly always possible in this way.

Dr. FrANk Smithies, Chicago: This is a practical method in the diagnosis and treatment of gastro-intestinal ailments. It seems quite impossible that apart from the diagnostic significance of these curves, an individual should fail to appreciate the importance that some of these curves carry with respect to treatment. One of the main points brought up during the past few years is the differentiation of gastric and duodenal ulcer. These curves enable us to differentiate quite easily the postpyloric from pyloric or antral ulcer. It seems that frequently it would be almost impossible in any other way to differentiate this class of case. Regarding the indications for treatment: There is no rule of thumls method if treatment of ulcer of the stomach or duodenum. There must be intense individualization of treatment. The treatment and the cure do not depend on anomalies of gastric secretion. Other factors come into play. The causes of ulcer indicate that in treatment we must first look to the individual factors in the production of ulcer. Afterward, if we give any medication, we must give it according to our knowledge of what the patient's stomach is doing. In many cases a great deal of medication is not needed. The work of Crohn bears out some of Dr. Rehfuss' observations, namely, that in the giving of alkali we very often cause more harm than good. We simply defeat nature in its attempt to secure in the stomach a restoration to normal.

With regard to the mucus cases Dr. Rehfuss mentioned: In these cases, where we have early secretion by the fractional method, within the first half hour or hour, and then have an accumulation, of a great deal of mucus, we are simply dealing with an instance where the gastric glands are easily fatigued, and the condition represents a form of gastric fatigue, with mucoid secretion instead of the specific secretion of that gland. We have seen it in patients who have been given great quantities of alkali. It is apparent from a knowledge of gastric physiology that what alkali does is simply to urge the stomach first of all to restore the natural reaction of the stomach contents, namely, faintly or definitely acicl. To do this it must overcome a foreign body, namely, the excess alkali. It does this by first producing a great deal of acid, then there follows physiologic fatigue, namely, an excessive secretion of mucus, which has to be washed out. If more alkali is given, in the end, the stomach does not produce acids. In periods varying from six to twelve days there is no acid secretion whatever. The glands are putting out a great quantity of mucus. This indicates mucoid degeneration, or physiologic fatigue of the cell.

Dr. Martin E. Rehruss, Philadelphia: Regarding Dr. Hayes' suggestion that this seems to be a little too extensive a procedure, a great majority of the men I have met do not think it is at all extensive. I do practically nothing else: My assistants are trained. I do not pass the stomach tube more than a few times a week. You can train nurses to do every little detail covering this work. It is a question of organization to do this work. It is absolutely impossible, by simple examination, to predict anything regarding the stomach. I have seen any number of people who were told that they had a normal stomach after a single examination.

Dr. Smithies mentioned glandular fatigue. We found that in the normal, healthy individual we can give a meal and develop a curve, and give a meal immediately after, developing the same curve, showing there was no glandular fatigue. However, we took students around the examination period, and not only found that we could produce physiologic fatigue, but also a form of nervous fatigue. We found a man who could not coagulate milk, and when he came back after a vacation the milk was beautifully curded, which shows the effect of giving it to him in that way.

The Asset of Good Health.-I am heartily in favor of this measure [bill for an addition to the Hygienic Laboratory], because it helps in the preservation of health. For years and years the medical men of the world have been trying to impress on the people this fact, that good health is the most valuable asset we possess. It is often said on the floor of this House that this war, as horrible as it is, will teach the American pcople many valuable lessons. And there is no doubt in my mind hut that one of the most valuable lessons that will be taught in this war will be the importance of preventing diseasc. Today we understand as we never understood before that it it necessary not only to preserve the health of our boys on the firing line, but it is also equally necessary to preserve the health of the people at home, who are united and working earnestly to produce the necessities for these boys on the firing line. I hope that the American people especially will never forget this lesson, and that from now on they will be willing to appropriate the money necessary, so that medical men can experiment and carry on the work that is necessary in order to prevent disease, and not wait until people are sick and then spend ten times more to be cured.Mr. Lazaro, Congressional Record, Oct. 2, 1918, p. 11044. 